\title{
La liberté du concept
}

\section{The Concept's Freedom}

RESUMEN: La primera edición de la Enciclopedia define la Lógica como la "ciencia de la libertad". Este estudio trata de explicar esta formulación investigando la concepción hegeliana del Sistema filosófico como un "círculo de círculos", la de la Lógica como una "Onto-logía", y luego la del Concepto como un autodesarrollo "subjetivo" del pensar "objetivo".

PALABRAS CLAVE: CONCEPTO; LIBERTAD; LÓGICA; MEDIACIÓN; SUBJETIVIDAD; SISTEMA.
ABSTRACT: The first edition of the Encyclopedia defines Logic as the «science of freedom». The paper intends to explain this formula by investigating Hegel's conception of the philosophical system as a 'circle of circles', that of Logic as an 'Onto-Logic', then that of the Concept as a 'subjective' self-development of 'objective' thinking. KEY WORDS: CONCEPT; FREEDOM; LOGIC; MEDIATION; SUBJECTIVITY; SYSTEM

\section{LE DISCOURS PHILOSOPHIQUE DE LA TOTALITÉ}

$\mathrm{L}$ 'ENCYCLOPÉDIE DES SCIENCES PHILOSOPHIQUES est l'exécution du programme, formulé par la Préface de la Phénoménologie de l'esprit et justifié par la Logique, d'un système de la science exposé du point de vue non de telle ou telle position subjective singulière (ce serait alors un système), mais de l'esprit saisi en son absoluité, comme processus dialectique de constitution de soi. Une telle science est véritablement une science de l'absolu, puisque l'absolu, dont l'esprit est « la définition la plus haute " ${ }^{1}$, s'y énonce pour lui-même selon ses

[1] Hegel, Enzyklopädie der philosophischen Wissenschaften [Enzyklopädie], §384 Anm., Gesammelte Werke [GW] 20, Hamburg, Meiner, 1992 p. 382 ; Encyclopédie des Sciences philosophiques, t. 3 [Encyclopédie 3], Paris, Vrin, 1988, p. 179. 
déclinaisons majeures : logique, nature, esprit. Voici comment l'Introduction de la deuxième édition de l'Encyclopédie décrit cette science :

La science de [l'absolu] est essentiellement système, parce que le vrai en tant que concret est seulement en tant qu'il se déploie en lui-même et se recueille et retient dans l'unité, c'est-à-dire en tant que totalité [...] Une démarche philosophique sans système ne peut rien être de scientifique; outre que pour elle-même une telle démarche philosophique exprime davantage une manière de penser subjective, elle est, suivant son contenu, contingente. Un contenu a seulement comme moment du Tout sa justification mais, en dehors de ce dernier, a une présupposition non fondée ou une certitude subjective; de nombreux écrits philosophiques se bornent à exprimer d'une telle façon seulement des manières de voir et des opinions. - Par système, on entend faussement une philosophie ayant un principe borné, différent d'autres principes; c'est au contraire le principe d'une philosophie vraie que de contenir en soi tous les principes particuliers. ${ }^{2}$

On peut tirer trois enseignements de ce passage. 1) Il n'y a de science, au sens fort du terme, que de la totalité du réel (ou plutôt de l'effectif); la philosophe seule peut revendiquer cette qualification, à condition toutefois de ne pas être une philosophie, ordonnée à un principe particulier, mais le système intégrant ces principes en un tout unitaire. 2) Seule une telle science philosophique de la totalité échappe au risque d'abstraction inhérent à toute démarche de pensée. Les sciences positives, si fécondes que soient leurs opérations, ne sont jamais des sciences au sens plénier du terme : non parce qu'elles sont positives - c'est au contraire la garantie de leur fécondité - mais parce qu'elles sont constitutivement « sans système », car ordonnées à un point de vue régional. C'est encore plus vrai des philosophies ordonnées à un "principe borné »; elles sont nécessairement des vues sur l'absolu, et non le savoir de l'absolu. 3) La systématicité consiste en ce que le tout du savoir découle d'un unique principe. Il n'y a donc, en toute rigueur, qu'une seule philosophie dont chaque philosophie particulière n'expose qu'un aspect : les Leçons sur l'histoire de la philosophie développent cette vue de manière conséquente en présentant la "philosophie Une " (die Eine Philosophie) comme le procès dont les philosophies particulières sont les moments, au sens à la fois logique et chronologique du terme. La systématicité n'est donc pas seulement une exigence du temps présent, elle est l'expression de la nature intemporelle d'un philosopher qui ne se donne pourtant à connaître que dans le temps, historiquement. D'où la thèse à première vue démentielle d'une correspondance entre les moments de l'idée logique et la succession des

[2] Hegel, Enzyklopädie, $\$ 14, G W$ 20, p. 56 ; Encyclopédie des Sciences philosophiques, t. 1 [Encyclopédie 1], Paris, Vrin, 1970, p. 180-181. 
systèmes philosophiques réduits à leur unique principe, d'une homologie de la philosophie et de l'histoire de la philosophie :

Je soutiens que la succession des systèmes philosophiques dans l'histoire est la même que la succession des déterminations conceptuelles de l'idée dans sa dérivation logique [...] L'histoire de la philosophie est la même chose que le système de la philosophie. ${ }^{3}$

La question se pose alors, bien entendu : faut-il identifier le système de Hegel à cette philosophie une et universelle dont les philosophies particulières ne sont que les moments ? La philosophie de Hegel est-elle la philosophie ? La réponse à cette question décisive est complexe. D’un côté, Hegel, en raison même des thèses qu'il professe quant à la nature du système, doit identifier sa pensée (qui de ce fait n'est pas sa pensée) à la science de l'absolu : c'est seulement lorsque l'on a accédé au point de vue de la totalité que l'on peut cerner la véritable signification de la systématicité. Mais, inversement, une telle vision de l'achèvement hégélien de la philosophie interromprait d'une manière forcément arbitraire le dynamisme processuel de la raison s'ex-posant dans l'histoire. On rencontre ici en son expression cruciale le dilemme du hégélianisme : il ne peut pas et pourtant ne peut que se penser comme ultime figure de la philosophie.

Quoi qu'il en soit, le système hégélien comporte une organisation originale, notamment par rapport au monisme de la Doctrine de la science fichtéenne et au dualisme méthodologique professé à Iéna par Schelling (la philosophie pouvant être exposée soit du point de vue subjectif de la philosophie transcendantale, soit du point de vue objectif de la Naturphilosophie ${ }^{4}$ ). Dès l'achèvement de la Phénoménologie de l'Esprit, la structure tripartite du système est acquise : il se compose de «la logique en tant que philosophie spéculative et [des] deux autres parties de la philosophie, les sciences de la nature et de l'esprit » ${ }^{5}$. La science philosophique se décompose donc en trois parties dont chacune est « un cercle se fermant sur lui-même " et expose néanmoins "l'Idée philosophique [...] dans une déterminité ou un élément particulier ${ }^{6}:$ la logique, "science de l'Idée en et pour soi », la philosophie de la nature, "science de l'idée en son

[3] Hegel, Leçons sur l'histoire de la philosophie. Introduction, trad. Marmasse, Paris, Vrin, 2004, p. 41 et 105.

[4] Voir Schelling, Système de l'idéalisme transcendantal, Préface, Louvain, Peteers, 1978, p. 000 (SW 3, p. 331) ; Exposition de mon système de la philosophie, Vrin, 2000, p. 33-34 (SW 4, p. 108).

[5] Hegel, «Selbstanzeige», in Phänomenologie des Geistes [PhG], édition WesselsClairmont, Meiner, 1988, p. $550 ; P h E$ J/L, p. 24.

[6] Hegel, Enzyklopädie, $\$ 15, G W$ 20, p. 56 ; Encyclopédie 1, p. 181. 
être-autre ", et la philosophie de l'esprit, où l'idée " de son être-autre, fait retour à soi-même ${ }^{7}$. Que signifie cette division inédite de la philosophie ?

La logique, telle que la conçoit Hegel, n’a pas d'équivalent dans la tradition. Elle prend la place, dit-il, de la métaphysique traditionnelle, donc de la " science de l'être en tant qu'être » ou, selon une dénomination plus récente, de l'ontologie ; le terme, comme on le sait, est dû à Wolff et s'applique à la métaphysique générale, distinguée de la métaphysique spéciale. Mais, en même temps, la logique est autre chose qu'une métaphysique ou qu'une ontologie, si l'on entend par là un discours portant sur un certain domaine d'objectivité, si éminent soit-il : elle prétend être une onto-logique, le logos même de l'être. On pourrait dire que la logique est la réflexion de l'être, le terme " réflexion " étant à entendre, en un sens non psychologique, comme libre retour sur luimême d'un double processus (celui de l'être et celui de la pensée) qui parvient seulement ainsi à se ressaisir en son unité fondamentale. La logique n'est donc certainement pas un " art de penser » ou un organon; elle présente plutôt, en leur liaison systématique, l'ensemble des conditions, ontiques aussi bien que noétiques, sous lesquelles la pensée de l'être peut se déployer ; elle développe ainsi une "pensée objective ${ }^{8}$.

La philosophie de la nature constitue la part maudite du système hégélien. Son discrédit s'explique d'abord par des circonstances générales (le désintérêt que l'on éprouve, à «l'âge de la science », pour ce qui apparaît comme une spéculation hasardeuse ; les aberrations de la Naturphilosophie romantique, dont Hegel s'est pourtant soigneusement démarqué) ; il est dû aussi à certaines erreurs commises par Hegel dans sa dissertation sur les orbites des planètes (et jamais désavouées), qui l'ont beaucoup desservi auprès des historiens des sciences et des scientifiques ${ }^{9}$. Pourtant, examiné sans prévention, le projet de la philosophie de la nature hégélienne mérite de retenir l'attention. Elle s'efforce en effet (c'est peut-être ce qui nous la rend si lointaine) de penser la nature en son unité, et non comme une collection rhapsodique de niveaux de réalité entre lesquels, malgré l'adage in natura non datur saltus, les sauts sont infranchissables. La nature en sa totalité se définit, on l'a vu, comme «l'être-autre de l'idée » et procède de la « décision » par laquelle l'idée logique « se congédie librement d'elle-même » ${ }^{10}$.

[7] Hegel, Enzyklopädie, $\$ 18, G W 20$, p. 60 ; Encyclopédie 1, p. 184.

[8] Hegel, Enzyklopädie, \$24 Anm., GW 20, p. 67 ; Encyclopédie 1, p. 290.

[9] Dominique Dubarle a écrit à propos de la critique hégélienne de Newton : « Le philosophe, honteux et confus, jura, mais un peu tard, qu'on ne l'y prendrait plus, et se fit épistémologue » (Préface à Hegel, Les orbites des planètes, Paris, Vrin, 1979, p. 15). Peut-on cependant dire, avec F. De Gandt, que Hegel « continue d'appartenir au même monde » que les Naturphilosophen romantiques ("Introduction », in Les orbites des planètes, p. 46) ? Ce n’est pas certain.

[10] Hegel, Wissenschaft der Logik, Band 2 [WdL 2], GW 12, p. 253 ; Science de la Logique, t. 3, trad. Jarczyk-Labarrière [SL 3 J-L], Paris, Kimé, 2014, p. 385, trad. mod. (Science 
Nombre de commentateurs ont perçu dans cette « libre décision » un coup de force grâce auquel Hegel aurait tenté de sortir du piège qu'il s'était tendu à luimême en faisant de l'idée absolue l'ultime catégorie non du système, mais de sa première partie, la Logique. Mais on peut considérer, à l'inverse, que cette libre aliénation du concept dans une nature classiquement comprise comme un mixte de nécessité et de contingence ${ }^{11}$ est ce qui permet de considérer celleci non comme un ensemble disparate, voire contradictoire, de phénomènes, mais comme un champ d'objectivité ouvert au concept. L'altérité radicale de la nature est, paradoxalement, ce qui autorise l'idée à se retrouver en elle. Elle fait signe, en d'autres termes, vers l'esprit.

Il faut mesurer ce qu'implique la position de l'esprit comme troisième terme récapitulatif : à l'encontre de tout spiritualisme, Hegel affirme que « pour nous, l'esprit a dans la nature sa présupposition $»^{12}$. C'est pourquoi la première section de la philosophie de l'esprit («Anthropologie»), montre comment la spiritualité émerge, de façon inconsciente, de la naturalité même : l'âme, que Hegel pense bien plus à la manière d'Aristote qu'à celle des modernes, est « le sommeil de l'esprit ${ }^{13}$, entendons : ce à partir de quoi il s'éveille à la conscience, puis à la rationalité. Hegel écrit que l'esprit est « la vérité existante de la matière » ${ }^{14}$.Paradoxalement, ce quasi-matérialisme lui paraît nécessaire si on veut éviter de faire de l'esprit une chose. Du même coup, selon un renversement caractéristique de la structure circulaire du savoir, l'esprit pose sa présupposition naturelle, dont il est « le [principe] absolument premier $" 15$. Une autre singularité de la philosophie hégélienne de l'esprit doit être soulignée : à côté de l'esprit subjectif (qui regroupe les multiples strates de la subjectivité, déjà étudiées d'un autre point de vue dans la Phénoménologie de l'Esprit) et de l'esprit absolu, qui est la pensée de soi de l'esprit, l'Encyclopédie accorde une large place aux expressions socio-politiques institutionnelles de la liberté « configurée en l'effectivité d'un monde ${ }^{16}$. Déjà, le chapitre VI de la Phénoménologie de l'Esprit traitait de l'incarnation historique de l'esprit en un "monde objectif effectif $»{ }^{17}$. L'adoption, à partir de 1817, de la dénomination inusitée d'esprit objectif souligne à quel

de la Logique, t. 3, trad. Bourgeois [SL 3 B], Paris, Vrin, 2016, p. 321).

[11] Voir Hegel, Enzyklopädie, $\$ 248, G W 20$, p. 237 ; Encyclopédie des Sciences philosophiques, t. 2 [Encyclopédie 2], Paris, Vrin, 2004, p. 187 : «la nature ne montre, dans son être-là, aucune liberté, mais de la nécessité et de la contingence ».

[12] Hegel, Enzyklopädie, \$381, GW 20, p. 381 ; Encyclopédie 3, p. 178.

[13] Hegel, Enzyklopädie, \$389, GW 20, p. 388 ; Encyclopédie 3, p. 185.

[14] Hegel, Enzyklopädie, \$389 Anm., GW 20, p. 389 ; Encyclopédie 3, p. 186.

[15] Hegel, Enzyklopädie, $\$ 381, G W 20$, p. 381 ; Encyclopédie 3, p. 178.

[16] Hegel, Enzyklopädie, $\$ 484, G W 20$, p. 478 ; Encyclopédie 3, p. 282.

[17] Hegel, Phänomenologie des Geistes [PhG], GW 9, Hamburg, Meiner, 1980, p. 238 ; Phénoménologie de l'Esprit, trad. Bourgeois [PhE B], Paris, Vrin, 2007, p. 384. 
point le concept hégélien d'esprit s'écarte de ce que l'on a entendu par là avant lui, et sans doute aussi après lui.

Il convient de réfléchir à la division du système en ces trois sphères, tout en sachant que " la représentation de la division a ceci d'incorrect qu'elle place les parties ou sciences particulières les unes à côté des autres, comme si elles étaient seulement des parties immobiles, [...] telles des espèces " ${ }^{18}$. En effet, chacune d'elles est à la fois close sur elle-même, donc susceptible d'un exposé autonome (comme celui des Principes de la philosophie du droit), et moment du tout unitaire qu'est le système. La solution de cette difficulté est formulée à la fin de l'Encyclopédie grâce à l'exposé des syllogismes correspondant aux trois lectures possibles de l'articulation interne du système. Point d'aboutissement de la philosophie de l'esprit, et plus précisément de l'esprit absolu, la philosophie, à savoir " l'idée qui se pense " ${ }^{19}$, se présente comme l'enchaînement (le système) de trois manifestations phénoménales, dans chacune desquelles l'un des trois moments - logique, nature, esprit - est en position de moyen terme. Dans le premier syllogisme, qui correspond à l'ordre d'exposition encyclopédique (L-N-E), « la médiation du concept a la forme extérieure du passage ${ }^{20}$, en sorte que « c'est seulement dans l'un des extrêmes [l'esprit] qu'est posée la liberté du concept $»^{21}$. Dans le deuxième syllogisme (N-E-L), c'est l'esprit qui est en position de moyen terme « qui présuppose la nature et l'enchaîne avec le logique ${ }^{22}$; cette seconde présentation décrit la manière dont le sujet peut penser son arrachement à la nécessité naturelle en direction de la liberté. Le troisième syllogisme (E-L-N) a pour moyen terme "la raison qui se sait » (la logique), et qui « se scinde en esprit et nature ${ }^{23}$; exposant la division de l'idée logique en un pôle subjectif (l'esprit) et un pôle objectif (la nature), il est à la fois la présupposition et le résultat du «jugement de soi » par lequel l'idée, comme totalité ou système, se décompose en deux " phénomènes » correspondant aux syllogismes précédents. Les commentateurs discutent encore de la position respective de ces trois syllogismes et de leur signification spécifique. En tout état de cause, il convient de noter qu'en présentant "l'idée de la philosophie » comme étant susceptible de trois lectures situées chacune sous la raison de l'un des moments de la totalité, Hegel offre une illustration saisissante, voire vertigineuse, de la circularité infinie du savoir spéculatif. Le système n'est pas réductible à un point de vue, fût-il, comme celui du Dieu leibnizien, celui de

[18] Hegel, Enzyklopädie, \$18 Anm., GW 20, p. 60 ; Encyclopédie 1, p. 184.

[19] Hegel, Enzyklopädie, \$574, GW 20, p. 569 ; Encyclopédie 3, p. 373.

[20] Hegel, Enzyklopädie, \$575, GW 20, p. 570 ; Encyclopédie 3, p. 373.

[21] Hegel, Enzyklopädie, \$575, GW 20, p. 570 ; Encyclopédie 3, p. 374.

[22] Hegel, Enzyklopädie, \$576, GW 20, p. 570 ; Encyclopédie 3, p. 374.

[23] Hegel, Enzyklopädie, $\$ 577, G W$ 20, p. 570 ; Encyclopédie 3, p. 374. 
la totalité : il est l'engendrement coordonné de tous les points de vue à travers lesquels « la nature de la Chose, le concept [...] poursuit son mouvement et se développe $»^{24}$.

\section{LA « SCIENCE DE LA LIBERTÉ »}

Paradoxe à l'aune des représentations communes : le système hégélien se présente comme l'expression même de la liberté. C'est parce qu'elle est systématique, organisée selon un certain lien de nécessité, que la philosophie mérite d'être définie comme la "science de la liberté ${ }^{25}$. Cette affirmation doit elle-même être entendue en toute sa complexité. Elle signifie d'abord que le système hégélien, qui n'est pas un cercle " clos en lui-même ${ }^{26}$, se prescrit d'accueillir la contingence en ce qu'elle a d'apparemment irréductible à la nécessité du concept. Rien n'est plus faux que la légende du nécessitarisme hégélien expulsant la contingence ou la réduisant à être l'ombre inconsciente d'une nécessité aveugle ; chez Hegel, il y a une réelle autonomie du contingent, car il est rationnel qu'il y ait de l'irrationnel ${ }^{27}$.

Mais la contingence n'est pas la liberté, et c'est pourquoi, à un deuxième niveau, il faut justifier la compatibilité, ou plus exactement le lien nécessaire de la nécessité et de la liberté. C'est ce que fait la Logique à l'occasion du passage, présenté comme « le plus dur » à concevoir ${ }^{28}$, de la " substance ", ultime catégorie de la Logique objective, au « concept » et à la Logique subjective :

Ainsi le concept est-il la vérité de la substance et, puisque le type de rapport déterminé de la substance est la nécessité, la liberté se montre comme la vérité de la nécessité et comme le type de rapport du concept. ${ }^{29}$

Ce que décrit cette transition de la substance au concept, ou de l'objectivité à la subjectivité, c'est précisément la constitution de la liberté à même la nécessité, c'est-à-dire le Beisichsein im Anderem, l'être «chez soi » du concept dans l'altérité du non-conceptuel ; la nécessité n'est pas supprimée par là, mais se trouve ordonnée à la libre processualité du concept s'engendrant lui-même

[24] Hegel, Enzyklopädie, $\$ 577, G W$ 20, p. 571 ; Encyclopédie 3, p. 374.

[25] Hegel, Enzyklopädie (1817), §5 Anm., GW 13, Hamburg, Meiner, 2000, p. 18 ; Encyclopédie 1, p. 156.

[26] Hegel, $P h G, G W$ 9, p 27 ; PhE B, p. 80.

[27] Voir B. Bourgeois, "Hegel et la déraison historique ", in Etudes hégéliennes, Paris, PUF, 1992, p. 271 sq. ; B. Mabille, Hegel. L'épreuve de la contingence, Paris, Aubier, 1999, en particulier section 7, p. $213 \mathrm{sq}$.

[28] Hegel, Enzyklopädie, \$159 Anm., GW 20, p. 175 ; Encyclopédie 1, p. 405.

[29] Hegel, $W d L$ 2, GW 12, p. 12 ; SL 3 J-L, p. 10 (SL 3 B, p. 16). Cf. Enzyklopädie, \$158, GW 20, p. 302 ; Encyclopédie 1, p. 403. 
ainsi que son autre. Mais si la liberté est la "vérité de la nécessité », et si cette dernière est la détermination la plus haute de la systématicité, alors le système lui-même doit être entendu non pas comme une totalité finie, mais comme une totalité en procès, comme dynamique de la vérité. Sans doute n'est-il pas exact de décrire le hégélianisme comme un système ouvert, si l'on entend par là qu'il serait indéfiniment révisable: il n'y a qu'un système, mais ce système est mobile, processuel, plastique si on veut. Ce qui manifeste le plus clairement cette propriété est le fait que, dans le système hégélien, les moments de totalisation sont aussi des moments d'immédiation : la totalité résultante qui récapitule le processus de déploiement (les médiations) d'un terme immédiat ou paraissant tel se constitue elle-même en une immédiateté seconde (une immédiateté devenue) qui résulte du processus conduisant à elle, mais aussi le légitime en retour, en sorte que le résultat (le second immédiat) est le fondement véritable du procès dont il résulte, et par conséquent du point de départ (le premier immédiat) de ce procès. On peut vérifier cette complémentarité de ce que j'appellerai la dialectique ascendante et la dialectique descendante en deux lieux stratégiques : la transition de l'idée logique à la nature à la fin de la Logique et le retour à la certitude sensible à la fin de la Phénoménologie.

Premier exemple. La circularité du savoir systématique, thématisée dans le chapitre sur l'idée absolue qui conclut la Logique, est la manifestation ultime de sa liberté, c'est-à-dire de sa capacité à engendrer par lui-même ses propres présuppositions et sa propre altérité : c'est pourquoi, au terme de la Logique, l'idée se donne librement congé pour se faire "idée dans la forme de l'être-autre ", autrement dit nature ${ }^{30}$. Donc, c'est dans la plus complète aliénation que l'idée logique trouve la vérification de sa liberté : le concept démontre sa puissance en s'ex-posant et en se reconnaissant dans un élément de radicale altérité. Mais un tel procès ne peut se clore en quelque point que ce soit, et c'est pourquoi la vérité du système hégélien se donne, à qui sait la déchiffrer, en chacun de ses points. L'absolu hégélien est, au sens étymologique, utopique : il ne réside nulle part, même si ses diverses expressions sont ordonnées par le mouvement du concept.

Deuxième exemple. Il n'y a d'immédiateté que devenue ou médiate : cette thèse finale de la Logique est, au fond, ce dont la conscience immédiate, dans la Phénoménologie de l'esprit, faisait déjà l'épreuve, mais de manière aveugle, en tant que certitude sensible. L'incapacité même de cette conscience à saisir de manière antéprédicative «ceci » en sa singularité absolue signifie qu'il n'est saisissable qu'à partir de l'autre de la conscience immédiate, le savoir absolu ; c'est bien pourquoi la certitude sensible ressurgit au terme du procès phénoménologique, où elle est alors présentée comme le résultat d'un " congé » que le savoir se donne, ; ce congé constitue même « la liberté suprême » du savoir

[30] Hegel, Enzyklopädie, § 247, GW 20, p. 237 ; Encyclopédie 2, p. 187. 
absolu que l'esprit a acquis de soi ${ }^{31}$. Le parallélisme est évident, jusque dans le vocabulaire du « congédiement » (Entlassung), entre le passage du savoir absolu à la conscience sensible et celui de l'idée absolue à l'être-autre naturel. En ces lieux stratégiques où le système paraît se recueillir en une expression ultime, où l'on croit parvenir à une dénomination définitive de l'absolu (puisque « les déterminations logiques en général peuvent être regardées comme des définitions de l'absolu » ${ }^{32}$ ), la circularité, image de la liberté de la processualité systématique, détruit l'illusion d'un point d'arrêt ou d'une ultime vérité. Soustrait à ce procès (celui-là même que saisit, selon la Préface de la Phénoménologie, la " proposition spéculative »), l'absolu lui-même ne serait " qu'une pensée visée ${ }^{33}$.

\section{LA LOGIQUE COMME DÉFINITION DU MODE SPÉCULATIF DE LA PENSÉE}

Au sein du « cercle de cercles » qu'est le système hégélien, une place fondamentale revient à la Logique. À bonne distance de l'utilisation usuelle de cette notion, la logique hégélienne se définit comme « la science de l'idée pure, c'està-dire de l'idée dans l'élément abstrait de la pensée »" ${ }^{34}$. Mais, précise Hegel dans ses leçons, de la pensée comme de la science on peut avoir « une haute ou une petite opinion ", et il est clair pour lui que ceux qui considèrent la logique comme une discipline seulement formelle en ont une petite : Kant, par exemple, pour qui la «logique générale» est " un canon de l'entendement et de la raison, mais seulement par rapport à ce qu'il y a de formel dans leur usage ${ }^{35}$. Pour sa part, la logique hégélienne n'est formelle qu'en un sens très particulier : par forme, il faut entendre ici le dynamisme immanent qui met en mouvement et dialectise « une matière pour qui la forme n'est plus un extérieur, puisque cette matière est bien plutôt la pensée pure, partant la forme absolue elle-même ${ }^{36}$. Ainsi, la logique est bien une science formelle, mais au sens où elle est la « science de la forme absolue qui au dedans de soi est totalité ${ }^{37}$. La logique hégélienne veut être une logique du mouvement («formel ») des significations (« matérielles »), donc une logique de la vérité : elle cherche à énoncer, d'une manière qui est encore "pure », c'est-à-dire ici abstraite, la configuration de l'être en sa totalité.

[31] Hegel, $P h G, G W$ 9, p. $432 ; P h E$ B, p. 660.

[32] Hegel, Enzyklopädie, § 85, GW 20, p. 121 ; Encyclopédie 1, p. 348

[33] Ibid.

[34] Hegel, Enzyklopädie, \$19, GW 20, p. 61 ; Encyclopédie 1, \$19, p. 283.

[35] Kant, Kritik der reinen Vernunft, B 77, ; Critique de la raison pure, trad. A. Renaut, GF-Flammarion, p. 814.

[36] Hegel, Wissenschaft der Logik, Band 1 [WdL $1^{1}$ ], GW 11, Hamburg, Meiner, 1978, p. 21 ; Science de la Logique, t. 1, trad. Jarczyk-Labarrière, Paris, Kimé, 2006 [SL 1 J-L], p. 18 (Science de la Logique, t. 1, trad. Bourgeois, Paris, Vrin, 2015 [SL 1 B], p. 57).

[37] Hegel, $W d L$ 2, $G W 12$, p. 25 et 27 ; SL 3 J-L, p. 25 et 27 (SL 3 B, p. 32 et 34). 
Cette Logique entend mettre en œuvre une forme de pensée qui « n’est pas seulement une pensée, mais bien plutôt [...] le mode le plus élevé selon lequel l'être éternel et qui est en et pour soi peut être saisi " ${ }^{38}$. Cette pensée spéculative doit être distinguée de la représentation, des savoirs d'entendement et de la pensée philosophique non spéculative. La Préface de la Phénoménologie de l'Esprit distingue ainsi la connaissance proprement dite (Erkenntnis) de la représentation (Vorstellung), rapport immédiat d'un sujet fini à un objet donné avec lequel elle procure tout au plus une certaine familiarité (Bekanntschaft) ; d'où la formule : "ce qui est bien connu (bekannt) est en général, pour cette raison qu'il est bien connu, non connu (erkannt) " ${ }^{39}$. La connaissance proprement dite résulte du travail de discrimination et de classification opéré par l'entendement, lequel manifeste sa " puissance absolue » en apprenant à l'esprit fini à « regarde[r] [l]e négatif en face », à « séjourne $[\mathrm{r}]$ auprès de lui » ${ }^{40}$. Il convient, pour éviter les simplifications, de conserver en mémoire ce vibrant éloge adressé par Hegel à l'entendement.

Mais il faut distinguer la pensée d'entendement, qui élève les représentations à une universalité abstraite, et le " penser pur ", qui a pour tâche de fluidifier les «pensées fixes » de l'entendement et de les transformer en concepts spéculatifs en épousant le mouvement même de ce qui est ${ }^{41}$. Surmonter le rapport d'extériorité qui paraît exister entre les onta et le logos, élever la conscience au point de vue spéculatif en décrivant les expériences qui rendent cette élévation nécessaire : tel est le but de cette introduction au savoir logico-spéculatif qu'est la Phénoménologie de l'Esprit. Toutefois, cette présentation des choses, justifiée du point de vue propédeutique qui est celui de l'ouvrage de 1807, semble faire de la science spéculative le résultat d'un processus de purification partant de la représentation sensible du donné en son immédiateté. Mais peut-on concevoir la science spéculative comme la simple clef rétrospective des expériences manquées de la conscience ? Ceci ne reviendrait-il pas à destituer le savoir logico-spéculatif de sa position absolue ? Pour prévenir ce risque, le «Concept préliminaire » de l'Encyclopédie présente la pensée logique comme la structure générative que présupposent aussi bien la simple représentation en laquelle « le contenu se tient isolé en sa singularité » que les actes intellectuels qui confèrent à cette représentation la forme de l'universalité en établissant « des relations de nécessité entre [ses] déterminations isolées ${ }^{42}$.

[38] Hegel, Enzyklopädie, $\$ 19$ Zusatz, W 8, p. 69 ; Encyclopédie 1, p. 469.

[39] Hegel, $P h G, G W$ 9, p. 26 ; $P h E$ B, p. 79.

[40] Hegel, $P h G, G W 9$, p. $27 ; P h E$ B, p. 80.

[41] Hegel, $P h G, G W$ 9, p. 28 ; $P h E$ B, p. 81.

[42] Hegel, Enzyklopädie, $\$ 20$ Anm., GW 20, p. 64 ; Encyclopédie 1, p. 286. 
Le penser spéculatif (das Denken) doit également être distingué de ce que Hegel qualifie de Nachdenken, c'est-à-dire de cette opération grâce à laquelle le sujet revient sur ses propres actes, concédant par là leur extériorité relative à son égard. Sans doute, ce re-penser, cette pensée d'après, est-il, pour le sujet empirique, le moyen d'accéder au "substantiel », car il entraîne « la refonte de l'immédiat ${ }^{43}$. Mais, du point de vue spéculatif, le Nachdenken présuppose le Denken, tout comme le fini présuppose l'infini qui est sa propre pulsation immanente. Les pensées au moyen desquelles l'esprit cherche à consigner la vérité des choses ne sont que les traces instantanées du mouvement du penser authentique. Elles relèvent de la pensée finie, une pensée qui demeure arrimée à des oppositions qui figent le processus fluide qui les engendre : sujet-objet, fini-infini, pensée-être, etc. D’une manière générale, toute structure de pensée dualiste relève de la "métaphysique d'entendement la plus ordinaire " ${ }^{44}$, et c'est la tâche de la spéculation de dissoudre ces oppositions qui structurent la représentation des choses, sa reprise réflexive et les concepts d'entendement qui en résultent en mettant à jour la dynamique qui les engendre. Comme l'idée logique, qui en est l'expression condensée, le penser spéculatif est « essentiellement processus ${ }^{45}$.

\section{LA LIBERTÉ DU CONCEPT}

Il est peu de termes auxquels Hegel a fait subir un tel déplacement de sens que celui de concept (Begriff). Conformément à son étymologie (concipere, be-greifen : saisir ensemble), le concept, traditionnellement, renvoie à l'unité d'une conscience ${ }^{46}$. Hegel, pour sa part, dissocie radicalement conscience et concept en faisant du concevoir l'acte par lequel la pensée s'engendre elle-même et produit par là son propre sujet en même temps que son objet. Le concept n'est pas l'œuvre d'un sujet concevant (fini), mais libre pensée de l'être par lui-même; il n'est pas le bien ou la chose d'un sujet, car on n'a pas des concepts comme on a un habit (la comparaison est de Hegel lui-même ${ }^{47}$ ), il est la subjectivité même, c'est-à-dire plus et autre chose qu'un simple « sujet grammatical » ${ }^{48}$ : la dynamique même de l'auto-engendrement de la pensée :

[43] Hegel, Enzyklopädie, $\$ 22$ Zusatz, Werke, éd. Moldenhauer-Michel, Band 8 [ $W$ 8], Frankfurt, Suhrkamp, 1970, p. 78 ; Encyclopédie 1, p. 473.

[44] Hegel, Enzyklopädie, $\$ 95$ Anm., GW 20, p. 132 ; Encyclopédie 1, p. 358.

[45] Hegel, Enzyklopädie, \$215, GW 20, p. 218 ; Encyclopédie 1, p. 449.

[46] Voir Kant, Kritik der reinen Vernunft, A 104 ; Critique de la Raison pure, p. 182 : le concept est « cette conscience une qui réunit en une représentation le divers intuitionné successivement $»$.

[47] Hegel, $W d L$ 2, GW 12, p. 17 ; SL 3 J-L, p. 16 (SL 3 B, p. 23).

[48] Hegel, $W d L$ 2, $G W 12$, p. 28 ; SL 3 J-L, p. 28 (SL 3 B, p. 35). 
Ce qui est à examiner ici, c'est le concept non pas comme acte de l'entendement conscient de soi, non pas l'entendement subjectif, mais le concept en soi et pour soi. $^{49}$

Le concept, si l'on peut dire, est le sujet émergent du procès du penser. De ce fait, on ne saurait le qualifier d'abstrait, en lui opposant le concret des choses ou de la vie : le concept est au contraire, bien qu'il comporte aussi un moment d'abstraction ("l'universel abstrait »), "l'absolument concret, le sujet comme tel ${ }^{50}$.

Comme celles de l'être (avec le passer, Übergehen) et de l'essence (avec le paraître, Scheinen), la logique du concept dispose d'un type de processualité spécifique : le développement (Entwicklung). Mais, à la différence de la logique de l'être, où la détermination provenait d'une limite extérieure, et de celle l'essence, où toute détermination était relative à une autre (comme l'intérieur à l'extérieur, ou l'essence elle-même par rapport à l'apparence ou à l'être), le concept tire de lui-même son être ou ses déterminations. Plus exactement, il redéploie à partir de soi-même la négativité extérieure qu'il a incorporée en " assimilant » (c'est-à-dire à la fois en intégrant et en " dépassant ») la logique objective, donc l'ensemble des déterminations de l'être et de l'essence. La vie organique offre une illustration de ce type de procès : le vivant, dirait-on aujourd'hui dans les termes de la théorie des systèmes, assimile l'information transmise par son environnement et la reformule selon son propre code. Le concept, la pensée, se développe donc à la manière d'un être vivant. Toutefois, on ne saurait dire que la théorie hégélienne du concept est une forme de vitalisme ou d'organicisme ; pour Hegel, contrairement à d'autres auteurs du $19^{\mathrm{e}}$ siècle, c'est la vie qui est concept, et non le concept qui est vie; c'est pourquoi il tient à distinguer la « vie logique », la vie du concept, de la « vie naturelle ${ }^{51}$.

Par ailleurs, le caractère « organique » du concept ne signifie pas que celuici soit pure affirmation d'identité, que le travail du négatif qui œuvrait dans la logique objective lui soit devenue étranger ; au contraire, «le concept est ce qui, comme négativité absolument identique à soi, se détermine soi-même ${ }^{52}$. Mais, dans la sphère du concept, le négatif n'est jamais donné ou présupposé, il est toujours suscité par le mouvement même du concept, lequel met en lumière «le développement de la négativité qu'il est déjà en soi » ${ }^{53}$. J'ai déjà évoqué l'exemple le plus remarquable de ce libre engendrement du négatif ou de l'altérité : lors-

[49] Hegel, $W d L$ 2, $G W$ 12, p. 20 ; SL 3 J-L, p. 19 (SL 3 B, p. 26).

[50] Hegel, Enzyklopädie, \$164 Anm., GW 20, p. 180 ; Encyclopédie 1, p. 411.

[51] Hegel, $W d L$ 2, $G W 12$, p. 180 ; SL 3 J-L, p. 276 (SL 3 B, p. 232).

[52] Hegel, $W d L$ 2, GW 12, p. 128 ; SL 3 J-L, p. 196 (SL 3 B, p. 168).

[53] Hegel, $W d L$ 2, GW 12, p. 61 ; SL 3 J-L, p. 91 (SL 3 B, p. 80). 
que, au terme de la logique, l'idée, expression achevée du concept, se donne librement congé à elle-même (sich frei entlässt) et s'abandonne à l'extériorité de la nature ${ }^{54}$. La liberté du concept consiste à se résoudre (sich entschliessen) à l'altérité et à se dissoudre dans l'altérité, à se livrer à la négation; c'est en ce sens qu'il est "puissance créatrice » ${ }^{55}$. L'arrière-plan théologique du propos est évident. Toutefois, il faut se garder de toute interprétation unilatéralement théologique : ce que les religions monothéistes entendent par Création est sans doute une illustration exemplaire, mais seulement une illustration, de la manière dont la totalité conceptuelle, en se développant, crée et libère des déterminités dont chacune est " comme un être libre du concept tout entier " ${ }^{56}$.

Comme on le sait, la doctrine du concept comporte trois sections qui traitent respectivement de la subjectivité, de l'objectivité et de l'idée. Il y a évidemment dans cette organisation quelque chose de paradoxal : pourquoi cette logique subjective qu'est la logique du concept traite-t-elle, plutôt que la logique objective qui la précède, de l'objectivité ? L'explication de ce paradoxe réside dans la radicalisation, chez Hegel, de la transformation des notions classiques de subjectivité et d'objectivité déjà entreprise par Fichte et Schelling dans leur discussion relative à la nature de l'idéalisme. Schelling introduit les dénominations d'idéalisme subjectif et objectif en 1795 dans les Lettres philosophiques sur le dogmatisme et le criticisme ${ }^{57}$; il les applique en 1801 respectivement à Fichte et à lui-même ${ }^{58}$. Fichte lui rétorque en 1804 que l'idéalisme n'a de sens qu'en tant que subjectivisme ${ }^{59}$. Je ne puis entrer dans le détail de cette controverse ; disons qu'il s'agit, pour ces philosophes, de dé-substantialiser la subjectivité et de la penser, comme dit Fichte, non pas comme Tatsache (fait), mais comme Tathandlung ( fait-acte ») ${ }^{60}$. Cela permet de comprendre que, chez Hegel, la logique du concept soit une théorie non subjectiviste de la subjectivité, réalisant le programme formulé par la Préface de la Phénoménologie de l'Esprit:

[54] Voir Hegel, $W d L$ 2, GW 12, p. 253 ; SL 3 J-L, p. 385 (SL 3 B, p. 321-322). - Enzyklopädie, $\$ 244, G W 20$, p. 392 ; Encyclopédie 1, p. 463.

[55] Hegel, $W d L$ 2, GW 12, p. 36 ; SL 3 J-L, p. 50 (SL 3 B, p. 47).

[56] Hegel, Enzyklopädie, \$ 161, GW 20, p. 177 ; Encyclopédie 1, p. 407.

[57] Schelling, Philosophische Briefe über Dogmatismus und Kriticismus, SW 1, 330 sq.; Premiers écrits, trad. Courtine, Paris, PUF, 1987, p. 202.

[58] Schelling, Darstellung meines Systems der Philosophie, SW 4, 109 ; Exposition de mon système de philosophie, trad. Catttin, Paris, Vrin, 2000, p. 34.

[59] Fichte, Wissenschaftslehre (1804), Werke 10, rééd. De Gruyter, 1971, p. 197-198 ; Doctrine de la Science (1804), trad. Julia, Paris, Vrin, 1967, p. 139-140.

[60] Voir Fichte, Zweite Einleitung in die Wissenschaftslehre, Werke 1, p. 459 ; Cuvres choisies de philosophie première, trad. Philonenko, Paris, Vrin, 1972, p. 269. 
« appréhender et exprimer le vrai comme substance, mais tout autant comme sujet ${ }^{61}$.

La sphère du concept est dans son ensemble celle de la subjectivité, au sens où le concept est le sujet qui émerge au cours de son auto-développement ou de son procès d'explicitation. Le " sujet " ainsi compris est donc bien la "vérité " (au sens hégélien de ce qui dépasse en l'assumant) de la "substance », ultime catégorie de la logique objective, tout comme la liberté est celle de la nécessité ${ }^{2}$. Le concept est, pour ainsi dire, la substance conçue comme n'étant rien d'autre que le procès de production de ses attributs, de ses modes et de ses accidents ; la doctrine hégélienne du concept est ainsi la contrepartie positive de l'explication avec la plus conséquente des philosophies de la substantialité, le spinozisme, qui se poursuit tout au long de la troisième section de la Doctrine de l'essence. La présence à soi du concept en chacune de ses déterminations successives Hegel dit de l'idée logique qu'elle est " parfaitement transparente à soi " ${ }^{63}$ - se manifeste de manière aiguë lorsque le concept subjectif se transpose dans l'objectivité, acquérant ainsi " une réalité», "un être » ${ }^{64}$. Mais, pour comprendre ce passage, il faut partir de la signification qu’a la subjectivité dans la première section de la logique du concept.

Si la subjectivité est la propriété distinctive du concept en tant que vérité processuelle de la substance, donc de la logique objective, elle est aussi, de façon plus étroite, celle du concept lorsqu'il est (provisoirement) séparé de l'objectivité qui lui fait face comme « la Chose même étant en soi et pour soi » ${ }^{65}$. Ce concept subjectif au sens étroit_ou encore formel, par opposition à matériel_s'explicite à travers un ensemble de formes classifiées, à la suite des Analytiques, par la logique scolastique : le concept proprement dit avec ses espèces (universel, particulier et singulier), ainsi que les types de jugements et de syllogismes concluants ou non. Ce concept formel apparaît " comme la sphère du simple entendement ${ }^{66}$, car il n'est - selon l'Ecole elle-même - qu'une "forme extérieure » s'appliquant à une matière passive. Mais Hegel s'écarte de manière décisive du formalisme de la logique post-aristotélicienne en restituant à la notion de forme, comprise comme pouvoir d'information, donc d'engendrement du réel, la signification dynamique qu'elle avait chez Aristote lui-même ${ }^{67}$ :

[61] Hegel, $P h G, G W 9$, p. $18 ; P h E$ B, p. 68.

[62] Voir Hegel, $W d L$ 2, $G W 12$, p. 12 ; SL 3 J-L, p. 10 (SL 3 B, p. 16).

[63] Hegel, $W d L$ 2, $G W 12$, p. 237 ; SL 3 J-L, p. 366 (SL 3 B, p. 300 ).

[64] Hegel, $W d L$ 2, GW 12, p. 125-126; SL 3 J-L, p. 180 (SL 3 B, p. 166).

[65] Hegel, $W d L$ 2, $G W 12$, p. 30 ; SL 3 J-L, p. 40 (SL 3 B, p. 37).

[66] Hegel, $W d L$ 2, $G W 12$, p. 30 ; SL 3 J-L, p. 40 (SL 3 B, p. 36).

[67] Sur l'information de la matière, voir Métaphysique H, 1042 a 24-1043 a 28, et le commentaire de Hegel : Vorlesungen über die Geschichte der Philosophie, W 19, p. 153 ; Leçons sur l'histoire de la philosophie, trad. Garniron, tome 3, Paris, Vrin, 1972, p. 518-519. 
Le concept est assurément à considérer comme forme, mais comme forme infinie, créatrice, qui renferme en elle-même et en même temps laisse aller hors d'elle la plénitude de tout contenu ${ }^{68}$.

Cette contestation du formalisme est particulièrement nette à propos du syllogisme. Bien qu'elle puisse apparaître comme une poursuite fastidieuse de la tradition scolastique, la théorie hégélienne du syllogisme (Schluss) fait de celui-ci l'acte par lequel le concept s'enchaîne à lui-même (schliesst sich mit sich zusammen). D'où l'identification du syllogisme et de la rationalité, laquelle ne saurait chez Hegel être de nature seulement formelle. Le syllogisme n'est donc pas une forme de raisonnement subjectif, mais bien plutôt le dynamisme immanent de la pensée de soi qu'est le « sujet », c'est-à-dire le concept :

Le syllogisme est le rationnel et tout rationnel [...] Le syllogisme rationnel consiste en ce que le sujet, par la médiation, s'enchaîne avec soi-même. C'est ainsi d'abord et seulement qu'il est sujet, ou encore le sujet est d'abord et seulement, en lui-même, le syllogisme de la raison ${ }^{69}$.

Pour résumer, la théorie du concept subjectif cherche à déprendre la subjectivité de tout contexte psychologique ou même transcendantal. La subjectivité désigne seulement le pouvoir qu'a la pensée de s'auto-déterminer : le sujet, c'est l'auto-mouvement du concept, la rationalité en acte de soi, et non pas l'origine présumée de cet acte. Si le concept subjectif est dit formel, ce n'est pas au sens où il rassemblerait un ensemble de formes susceptibles de s'appliquer à une matière indifférente, mais plutôt au sens où cette médiation de soi par soi qu'est le concept doit informer le réel, mieux : s'éprouver comme le mouvement même du réel. La subjectivité, dès lors, est seulement la médiation conceptuelle qui constitue l'objectivité comme objectivité.

L’objectivité, qui résulte de l'auto-suppression de la médiation de soi par soi qu'est le concept subjectif, est l'ultime figure de l'immédiateté dans la Logique; elle en récapitule donc les précédentes figures : l'être-là, l'existence, l'effectivité ${ }^{70}$. Mais l'objet, comme tout immédiat, est pénétré de médiation (conceptuelle). Autrement dit, c'est parce que le concept est subjectif qu'il y a de l'objectivité non pas " pour » un sujet, mais par la médiation (en apparence abolie dans son résultat) de la subjectivité. Ramassé en lui-même, ayant effacé la médiation conceptuelle qui le porte, l'objet est « l'être étant en et pour soi du concept qui a aboli en relation immédiate à soi-même la médiation posée

[68] Hegel, Enzyklopädie, $\$ 160$ Zusatz, W 8, p. 306 ; Encyclopédie 1, p. 590.

[69] Hegel, Enzyklopädie, \$ 181-182, GW 20, p. 191-193; Encyclopédie 1, p. 422-423.

[70] Hegel, $W d L$ 2, GW 12, p. 130 ; SL 3 J-L, p. 198-199 (SL 3 B, p. 171). 
dans son auto-détermination ${ }^{71}$. C'est une des particularités remarquables de la logique du concept : la médiation (subjective) y précède l'immédiat (l'objet), et celui-ci ne peut être pensé que comme immédiateté devenue, toujours déjà médiatisée. La logique objective révélait progressivement la médiation (essentielle) à l'œuvre dans l'immédiat apparent (l'être ou l'être-là " donné »); au contraire, la théorie de l'objectivité fait surgir, ou plutôt laisse se révéler les médiations conceptuelles (subjectives) que l'objet a pour ainsi dire « oubliées ». Du mécanisme à la téléologie, en passant par le chimisme, elle consiste en une anamnèse (Er-innerung) de la médiation conceptuelle, en une remémoration de l'être toujours déjà médiatisé de l'immédiat.

Comme pour la subjectivité, Hegel s'écarte de la conception commune de l'objectivité et de l'objet. En premier lieu, l'objet est objet en et pour lui-même et non pas d'abord par et pour un sujet : ce n'est que de manière secondaire que l'objet (Objekt) est un ob-jet (Gegenstand) se tenant en face d'un sujet :

Que l'objet soit aussi ob-jet et quelque chose d'extérieur à autre chose, cela va se déterminer par la suite, dans la mesure où il se pose dans l'opposition au subjectif; ici, tout d'abord, en tant qu'il est ce en quoi le concept est passé en sortant de sa médiation, il est seulement objet immédiat, naïvement là ${ }^{72}$.

C'est lorsque l'objet, dans l'étude de la téléologie, apparaît ordonné à des fins subjectives, que l'activité du sujet va émerger de nouveau au sein de l'objectivité, mais comme ce qui doit s'auto-dépasser en se réalisant : réalisé, le but subjectif n'est précisément plus subjectif mais «unité posée du subjectif et de l'objectif ${ }^{73}$. Hegel entend ainsi surmonter l'opposition entre finalité externe et interne : même si c'est « un des plus grands mérites de Kant » que d'avoir fait cette distinction, il convient de remonter, au-delà d'elle, jusqu'à ce qui constitue «l'essentiel du téléologique » : « la forme de la finalité », qui est conceptuelle ${ }^{74}$.

Par ailleurs, du fait même qu'elle résulte d'une Aufhebung du concept en tant que subjectif, l'objectivité est d'emblée conceptuelle : «l'objectivité est le concept réel, sorti de son intériorité et passé dans l'être-là " ${ }^{75}$. Se comprend ainsi l'intérêt qu'éprouve Hegel pour la preuve ontologique de l'existence de Dieu dans sa forme anselmienne ou cartésienne, malgré la critique radicale à laquelle Kant l'a soumise ${ }^{76}$. En effet, au-delà de sa teneur théologique, l'argument

[71] Hegel, $W d L$ 2, GW 12, p. 131 ; SL 3 J-L, p. 200 (SL 3 B, p. 173).

[72] Hegel, Enzyklopädie, \$193 Anm., GW 20, p. 200 ; Encyclopédie 1, p. 431.

[73] Hegel, Enzyklopädie, \$210, GW 20, p. 213 ; Encyclopédie 1, p. 445.

[74] Hegel, $W d L$ 2, GW 12, p. 156-157 ; SL 3 J-L, p. 240 (SL 3 B, p. 205).

[75] Hegel, $W d L$ 2, $G W$ 12, p. 30 ; SL 3 J-L, p. 40 (SL 3 B, p. 37).

[76] Voir Kant, Kritik der reinen Vernunft, A 592/B 620 sq. : Critique de la raison pure, p. $530 s q$. 
anselmien est l'exemple parfait du passage du concept à l'objectivité, ou encore de l'identité en procès du concept et de l'être : dans les deux cas, « le concept [...] se pose comme un réel, un étant $"{ }^{77}$. Ainsi comprise, la preuve ontologique est une illustration du propos général de la logique hégélienne ; c'est pourquoi, dans ses leçons de 1829 sur les preuves de l'existence de Dieu, Hegel dit de la Logique qu'elle est "la théologie métaphysique ", l'unique preuve de la vérité de la représentation religieuse ${ }^{78}$.

La refonte des concepts de subjectivité et d'objectivité procède d'un unique dessein : il s'agit de montrer que la subjectivité n'est pas une forme séparée et que l'objectivité n'est pas une matière informe. Si l'on ne pense pas l'objectivité comme intrinsèquement conceptuelle, on condamne le concept à n'être que le vêtement d'une subjectivité vide, coupée des choses : "en soi concept - ou encore, si l'on veut, subjectivité - et objet sont la même chose ${ }^{79}$.

L’idée, qui n'est pas un état (et surtout pas un état mental !) mais un processus, est l'explicitation de l'identité demeurée jusqu'alors implicite de l'objectivité et de la subjectivité, de l'être et du concept, de l'effectivité et de la rationalité, dont la "vérité est seulement celle d'être des moments de l'idée " ${ }^{80}$. On peut donc définir l'idée comme une totalité dynamique qui engendre et abolit par elle-même tous les moments et toutes les oppositions qui ont surgi du parcours entier de la Logique. L'idée au sens hégélien est ainsi un rempart contre le dualisme, non seulement parce qu'elle récuse les dualités que l'entendement considère comme ultimes, mais parce qu'elle est la règle immanente de constitution et de déconstruction de ces dualités.

De même que le concept n'est pas l'œuvre d'un sujet, mais la subjectivité même, l'idée n'est pas l'idée de quelqu'un sur quelque chose ; les deux premières sections de la logique du concept ont définitivement récusé une telle manière de voir. L'idée est " sujet-objet " ${ }^{81}$ au sens où elle est idée de soi, acte de soi : elle n'est rien d'autre que le procès grâce auquel le réel et la pensée s'engendrent simultanément, le procès qui institue l'être comme concept et le concept comme être. Elle résume ainsi le propos de la Logique comme onto-logique. Il faut donc admettre que le hégélianisme est loin de professer un réalisme de l'idée ; car c'est autre chose de dire que l'idée est la seule réalité véritable et de prétendre, comme Hegel, que l'idée est l'idée $d u$ réel. En ce sens, en dépit de la déclarations

[77] Hegel, $W d L$ 2, GW 12, p. 128 ; SL 3 J-L, p. 196 (SL 3 B, p. 168).

[78] Hegel, Leçons sur les preuves de l'existence de Dieu, trad. Lardic, Paris, Aubier, 1994, p. 111.

[79] Hegel, Enzyklopädie, $\$ 193$ Anm., GW 20, p. 202 ; Encyclopédie 1, p.432.

[80] Hegel, Enzyklopädie, $\$ 213$ Zusatz, W 8, p. 369 ; Encyclopédie 1, p. 616.

[81] Hegel, $W d L$ 2, $G W 12$, p. 176 ; SL 3 J-L, p. 269 (SL 3 B, p. 229). 
de Hegel selon laquelle "toute philosophie véritable est un idéalisme " ${ }^{82}$, sa philosophie n'est un idéalisme qu'un sens très particulier, puisque l'idée telle qu'il la définit est «l'unité de l'idéel et du réel ${ }^{83}:$ l'idéalisme hégélien conjoint la radicalisation de l'idéalisme (rien n'est réel sinon l'idée) et celle du réalisme (l'idée n'est rien si elle n'est pas) ${ }^{84}$.

Il faut alors se garder de commettre à propos de l'idée absolue, point d'aboutissement de la Logique (mais non du Système), un contresens comparable à celui qui est souvent commis à propos du savoir absolu de la Phénoménologie de l'esprit. L'idée absolue n'est pas l'ultime récapitulation de ce qui s'est accumulé tout au long du procès logique, car le terme ultime n'est rien s'il est coupé du procès qui y conduit : «l'intérêt réside dans le mouvement tout entier " ${ }^{85}$. L'idée absolue n'a pas d'autre contenu que celui qui peut se lire en chacun des moments du procès logique, depuis les catégories les plus pauvres («l'être pur », le «néant pur ») jusqu'aux plus complexes (le syllogisme, la téléologie). Mais elle le dit sous l'angle de son caractère processuel : elle « est, à cet égard, à comparer au vieillard qui prononce les mêmes assertions religieuses que l'enfant, mais pour qui celles-ci ont la signification de toute sa vie ${ }^{86}$. "Vieillesse » de la pensée, l'idée absolue en résume la vie, c'est-à-dire la liberté.

[82] Voir Hegel, Enzyklopädie, \$95 Anm., GW 20, p. 133 ; Encyclopédie 1, p. 360.

[83] Hegel, Enzyklopädie, \$214 Anm., GW 20, p. 217 ; Encyclopédie 1, p. 449.

[84] Voir O. Tinland, L'idéalisme hégélien, Paris, CNRS Editions, 2013.

[85] Hegel, Enzyklopädie, \$237 Zusatz, W 8, p. 370 ; Encyclopédie 1, p. 623.

[86] Ibid. 